\title{
Correction to: Carotid Atherosclerosis in Patients with Atrial Fibrillation
}

\section{Zhaojia Wang $^{1} \cdot$ Panagiotis Korantzopoulos $^{2} \cdot$ Tong Liu $^{1}$}

Published online: 10 December 2019

(C) Springer Science+Business Media, LLC, part of Springer Nature 2019

\section{Correction to: Current Atherosclerosis Reports (2019)} https://doi.org/10.1007/s11883-019-0808-4

Due to typesetting mistake, the image of Fig. 1 got corrupted.

The original version has been corrected.

$1 \quad$ Tianjin Key Laboratory of Ionic-Molecular Function of Cardiovascular Disease, Department of Cardiology, Tianjin Institute of Cardiology, The Second Hospital of Tianjin Medical University, Tianjin, China

2 First Department of Cardiology, Medical School, University of Ioannina, Ioannina, Greece 